\title{
Separation of pulsar signals from noise using supervised machine learning algorithms
}

\author{
Suryarao Bethapudi ${ }^{\mathrm{a}}$, Shantanu Desai ${ }^{\mathrm{a}}$ \\ ${ }^{a}$ Department of Physics, IIT Hyderabad, Kandi, Telangana-502285, India
}

\begin{abstract}
We evaluate the performance of four different machine learning (ML) algorithms: an Artificial Neural Network Multi-Layer Perceptron (ANN MLP), Adaboost, Gradient Boosting Classifier (GBC), and XGBoost, for the separation of pulsars from radio frequency interference (RFI) and other sources of noise, using a dataset obtained from the post-processing of a pulsar search pipeline. This dataset was previously used for the cross-validation of the SP INN-based machine learning engine, obtained from the reprocessing of the HTRU-S survey data (Morello et al., 2014). We have used the Synthetic Minority Over-sampling Technique (SMOTE) to deal with high-class imbalance in the dataset. We report a variety of quality scores from all four of these algorithms on both the non-SMOTE and SMOTE datasets. For all the above ML methods, we report high accuracy and G-mean for both the non-SMOTE and SMOTE cases. We study the feature importances using Adaboost, GBC, and XGBoost and also from the minimum Redundancy Maximum Relevance approach to report algorithm-agnostic feature ranking. From these methods, we find that the signal to noise of the folded profile to be the best feature. We find that all the ML algorithms report FPRs about an order of magnitude lower than the corresponding FPRs obtained in Morello et al. (2014), for the same recall value.
\end{abstract}

Keywords: methods: data analysis stars: neutron

\section{INTRODUCTION}

Ever since the accidental discovery of 'The Little Green Man' (Hewish et al., 1968) fifty years ago, a massive amount of work force and computing resources have been invested in exploring the observable Universe to detect radio pulsars. Pulsars are highly magnetized rotating neutron stars, with misaligned magnetic and rotation axes, which emit pulsed radio emission. Their radio signals are observable when the axis of their emission cone is directed along the line of sight to the observer. Subsequently, they have also been observed throughout the electromagnetic spectrum. An updated review of the various observational properties of pulsars and other kinds of neutron stars can be found in Kaspi and Kramer (2016).

Pulsars have provided a remarkable laboratory for tests and applications of nearly all branches of physics, from condensed matter physics to quantum chromodynamics, and also on a wide variety of topics in astrophysics spanning stellar evolution, interstellar medium, cosmology etc. (Blandford, 1992; Ransom, 2013). They can also be used to provide insights into the nature and distribution of dark matter (Baghram et al., 2011; Desai and Kahya, 2016). Pulsar observations led to the first confirmed discovery of extrasolar planets (Wolszczan and Frail, 1992), provided the first indirect evidence for gravitational waves (Taylor, 1994), and could also provide evidence for the direct detection of gravitational waves in the $\mathrm{nHz}$ regime (Detweiler, 1979). Therefore, it is imperative to discover new pulsars in order to harness their tremendous physics potential.

Email addresses: ep14btech11008@ith.ac.in (Suryarao Bethapudi), shantanudeiith.ac. in (Shantanu Desai)
As of April 2017, there are 2613 known pulsars in the ATNF catalog (Manchester et al., 2005). However, the total observable pulsar population in our galaxy has been estimated to be from 20,000 to $10^{5}$ (Yusifov and Küçük, 2004; Faucher-Giguère and Kaspi, 2006; Johnston and Karastergiou, 2017). If these estimates are correct, $\sim 90 \%$ of the Galactic pulsar population is yet to be discovered.

Pulsar searches from modern radio surveys involve sifting through candidates detected by pulsar search pipelines, consisting of either periodicity or single-pulse searches. These pulsar search algorithms are often computationally very expensive (although improvements continue to be made to their speed and sensitivity e.g., Smith 2016; Cameron et al. 2017). The output of these pipelines yield millions of candidates, out of which a small fraction consists of pulsars and the remaining candidates arise from radio frequency interference (RFI) or other sources of noise (Keith et al., 2010). Many of these candidates are visually inspected and manually vetted by domain experts. For current generation pulsar surveys, it takes about 1-300 seconds to vet each candidate (Eatough et al., 2010). Therefore, it would require up to 80,000 person hours to visually vet the million or so candidates. Such a manual visual classification of the pulsar candidates becomes intractable during the SKA era, where we expect to discover around 20,000 new pulsars (Kramer and Stappers, 2015). Even though the sifting of real pulsar signals from noise can be facilitated with graphical utilities such as JREAPER (Keith et al., 2009), these have limitations and one is prone to make mistakes (Bates et al., 2012; Eatough et al., 2010). Therefore, to maximize the detection of pulsars in the SKA era, the computational costs during all the steps of the pulsar search pipeline should be reduced and human intervention 
should be minimized at every step. An important step in this process would be to automate the filtering of pulsar candidates obtained from pulsar search pipelines as much as possible.

Therefore, for the autonomous identification of true signals from noise, the radio pulsar community has resorted to machine learning to solve this problem (Eatough et al., 2010; Bates et al., 2012; Lyon et al., 2013; Lyon et al., 2014; Morello et al., 2014; Lyon et al., 2016; Zhu et al., 2014; Wagstaff et al., 2016; Devine et al., 2016). In most of these papers, the machine learning algorithm used is some variant of an Artificial Neural Network (ANN). Supervised machine learning using an ANN was first used in the pulsar community by Eatough et al. (2010) to process 16 million pulsar candidates obtained by reprocessing data from the Parkes multi-beam survey. Bates et al. (2012) also used an ANN in the data-processing pipeline for the High Time Resolution Universe (HTRU) mid-latitude survey. They were able to reject $99 \%$ of the noise candidates and detect $85 \%$ of the pulsars through a blind analysis. Zhu et al. (2014) used a combination of three different supervised algorithms, namely an ANN, Support Vector Machine (SVM), and Convolution Neural Nets in their image recognition based post-processing pipeline dubbed PICS (Pulsar Image-based Classification System) AI. PICS was trained with data from the Pulsar Arecibo L-band Feed Array (PALFA) survey and validated with data from the Green Bank North Celestial Cap survey (GBNCC). From the validation set, PALFA was able to rank $100 \%$ of the pulsars in the top $1 \%$ of all candidates, while $80 \%$ were ranked higher than any noise or interference events. Lyon et al. (2013) studied the performance of various stream classifiers, such as very fast decision trees on pulsar data from the HTRU survey. They demonstrated the susceptibility of the pulsar data to the imbalanced learning problem and how the imbalance severely reduces pulsar recall. Thereafter, Lyon et al. (2014) presented a new classification algorithm for imbalanced data streams using Hellinger distance measure, which they applied to pulsar data from the HTRU survey. They were able to demonstrate that the algorithm can effectively improve minority class recall rates on imbalanced data. Morello et al. (2014) used neural networks in a pulsar ranking pipeline dubbed Straightforward Pulsar Identification using Neural Networks (SPINN). This pipeline was able to identify all the pulsars in the HTRU-S survey with a false positive rate of $0.64 \%$ and also helped reduce the amount of candidates to scan by up to four orders of magnitude. In this work, we apply multiple machine learning algorithms to the same dataset, as the candidates used for the cross-validation of the SPINN pipeline were made publicly available ${ }^{1}$. Lyon et al. (2016) presented a new method for on-line filtering of pulsars from noise candidates using a tree-based machine learning classifier called Gaussian Hellinger Very Fast Decision Tree. This algorithm was able to process millions of candidates in seconds and had recall rates of close to $98 \%$, when applied to data from the HTRU-1 and LOTAAS surveys with recall rates $>90 \%$ and false positive rates $<0.5 \%$. It also helped discover about 20 new pulsars from the LOTAAS survey (Lyon et al.,

${ }^{1}$ http://astronomy. swin.edu. au/ vmorello/
2016). Wagstaff et al. (2016) incorporated a machine learning based classifier, based on random forests in their radio transient detection pipeline, called V-FASTR. This was able to automatically filter out known event types (pulsars and artifacts) with an accuracy of $98.6 \%$ and achieved a $99-100 \%$ accuracy on newly collected data. Most recently, Devine et al. (2016) used six different machine learning algorithms to classify dispersed pulsar groups in the second stage of their single-pulse search pipeline, which is also sensitive to other "siblings" of radio pulsars, such as Rotating Radio Transients (RRATs) (McLaughlin et al., 2006) and Fast Radio Bursts (FRBs) (Lorimer et al., 2007) These six algorithms included an ANN (Bishop, 2006), SVM (Cortes and Vapnik, 1995), direct rule learner (Cohen, 1995), standard tree rule learner (Salzberg, 1994), hybrid ruleand-tree learner (Frank and Witten, 1998), and Random Forests (Breiman, 2001). Their benchmark dataset consisted of over 300 pulsar signals and about 9,600 noise candidates using observations from the Green Bank Telescope. They found that multiclass ensemble tree learners were the most efficient. Therefore, in most of the above papers, some variant of an ANN has been used for the post-processing of candidates from the pulsar search pipelines. Here, we start out with an ANN and showcase other algorithms which perform as good as or better than an ANN.

The outline of this paper is as follows. In Section 2, we provide an abridged pedagogic introduction to machine learning, including the definition of various quality scores. The pulsar dataset from the HTRU-S survey along with the features used for training are described in Section 3. In Section 4, we list the various machine learning algorithms applied to the pulsar dataset. Our implementation of ANN, Adaboost, Gradient Boosting Classifier, and XGBoost are described in Sections 4.1, 4.2, 4.3, and 4.4 respectively. We discuss the feature selection procedure in Section 5. We report the results of our machine learning algorithms in Section 6. We then conclude in Section 7.

\section{SUPERVISED MACHINE LEARNING TERMINOL- OGY}

There are two types of supervised machine learning problems, namely classification and regression. Given a collection of samples, where each sample uniquely belongs to a group (also known as it's class), classification is the process of putting samples into their respective classes as accurately as possible. Each sample is described via a collection of variables called 'features', which in some way is representative of the characteristics of each class. If for a given collection of samples, its true class labels (used to denote class membership) are also known, it is possible to build a mathematical model, which maps the 'features' (input) of a sample to the predicted class label (i.e., the class to which a sample belongs to) with high accuracy. A collection of samples for which both the feature data and class labels are known, is referred to as "labeled data". Labeled data is usually bifurcated into two distinct sets: i) a 'training set' used to 'learn' a function that maps a sample's features to a 
label, and ii) a test set used to assess the classification performance. A special case of classification involving only two classes is called binary classification. The separation of real pulsar signals from noise is an example of binary classification. The relationship between the features and their class variables is determined using a training set, which is a subset of the data for which we know both the features and the class labels.

It is a commonplace to use cross-validation to split randomly the available data into training and testing folds (independent samples) for analysis, to avoid problems associated with over-fitting or under-fitting, which leads to sub-optimal performance in practice. Cross-validation is used to build and test classification models iteratively, yielding an indication of aggregate data set performance (i.e. test on fold 1, train on remaining folds, then test on fold 2 and so on, aggregate results). Cross-validation is thus important for checking that the models we build are capable of generalizing beyond small training sets. The performance on the test set is used as the criterion to select the best algorithm.

More details on supervised machine learning techniques can be found in various monographs (Bishop, 2006; Michalski et al., 2013) and their astronomical applications are reviewed in (Ball and Brunner, 2010; Cavuoti, 2013).

\subsection{Evaluation measures}

In order to comparatively rank the performance of various machine learning algorithms, we calculate various performance scores for each algorithm, which we define in this section. We have used eight different scores $^{2}$ to quantify the model performance, viz. recall, precision, accuracy, $f_{1}$ score, log loss, G-Mean, Area under Receiver Operating Characteristics (AuROC) and False Positive Rate (FPR).

In addition to these eight scores, we also evaluate the confusion matrix for every algorithm. A confusion matrix is a $c \times c$ matrix, where $c$ indicates the number of class labels. Each element $\left(C_{j k}\right)$ of a confusion matrix is the number of observations labeled by the classifier as $j$, but which belong to class $k$. For our binary classification problem, the target class is considered to be positive if the candidate being classified is a pulsar and assigned the label of one. If the target class is a noise or RFI candidate, the candidate is assigned a negative class with label equal to 0 or -1 . In the case of binary classification problem (our case), each element of the confusion matrix corresponds to TP, TN, FP, and FN, which are defined in Table 1. In a multi-class classification setting, such a correspondence is absent.

1. TP: True Positive. Those observations belonging to the TRUE class, which are correctly labeled by the classifier as positive.

2. TN: True Negative. Those observations belonging to the FALSE class, which are correctly labeled by the classifier as negative.

\footnotetext{
${ }^{2}$ Score is a floating point number, which quantifies the performance of an algorithm
}

\begin{tabular}{ccc}
\hline & POSITIVE & NEGATIVE \\
\hline TRUE & TP & TN \\
FALSE & FP & FN \\
\hline
\end{tabular}

Table 1: Confusion matrix for a binary classification problem. The rows correspond to the ground truth labels and the columns correspond to the labels as assigned by the classifier. Definitions of the symbols used here can be found in Section 2.1

3. FP: False Positive. Those observations belonging to the FALSE class, which are incorrectly labeled by the classifier as positive. Hence, the name False Positive. This type of error is also called Type-I error.

4. FN: False Negative. Those observations belonging to the TRUE class, which are incorrectly labeled by the classifier as negative. This is also referred to as Type-II error.

Now, we define each of the eight scores used to evaluate the algorithms used in this paper.

Recall (also known as Sensitivity) is defined as,

$$
\frac{T P}{T P+F N} \text {. }
$$

A pristine machine learning classifier will have a recall value of 1 and the minimum value for an egregious classifier would be 0 . A Recall of one implies that all positively classified observations belong to the TRUE class. This score is given prominence, when misclassifying a TRUE class observation has a higher cost than misclassifying a FALSE class observation, or in other words when Type-II error has a higher penalty than Type-I error. This is the case we need to consider for the pulsar separation problem discussed in this work.

Precision is defined as,

$$
\frac{T P}{T P+F P} \text {. }
$$

Precision is the ratio of TRUE class observations labeled correctly to the total number of observations, which were labeled as positive (irrespective of whether they are true positives or not). Similar to recall, even here the best value is 1 and worst value is 0 . Higher the value of this score, better is the classifier in ascertaining the TRUE class observations.

Accuracy is the most popular score in any classification scenario, where the class distributions are equally balanced, and is defined as,

$$
\frac{T P+T N}{T P+T N+F P+F N} .
$$

This score has the value of 1 as the best value and 0 as the worst. An accuracy of 1 implies that the classifier isn't making any error whatsoever. 
$f_{1}$ score also known as F-measure or balanced Fscore (Bishop, 2006) is defined as,

$$
\frac{2 \times \text { Precision } \times \text { Recall }}{\text { Precision }+ \text { Recall }} \text {. }
$$

In other words, $f_{1}$ score is the harmonic mean of precision and recall. It provides an alternate measure of accuracy and has the best value at 1 and worst value at 0 . We note that precision, recall, and accuracy range between values of 0 and 1, where a value of 1 indicates the absence of errors for that score.

Log Loss also known as logistic loss or cross-entropy loss is defined as,

$$
\log \operatorname{loss}=-\frac{1}{\mathrm{~N}} \sum_{\mathrm{i}=1}^{\mathrm{N}}\left(\mathrm{y}_{\mathrm{i}} \log \left(\mathrm{p}_{\mathrm{i}}\right)+\left(1-\mathrm{y}_{\mathrm{i}}\right) \log \left(1-\mathrm{p}_{\mathrm{i}}\right)\right) .
$$

In Eqn. 5, $y_{i}$ represent the class labels and $p_{i}$ are the corresponding probabilities. Unlike the other scores discussed above, the range of values of logloss falls between $[0, \infty)$, where a score of 0 is achieved in the case of a perfect classifier. Interested readers are advised to refer to Bishop (2006) for more details.

G-Mean is the geometric mean of recall and specificity. It is defined as,

$$
\sqrt{\text { Recall } \times \text { Specificity }} \text {. }
$$

Specificity (Fawcett, 2006) is a measure of how well the classifier is able to label a negative class. It is analogous to recall, which is a measure of how well the classifier is able to label a positive class. The best and worst values of G-Mean score are 1 and 0 respectively. This score has been given prominence in unbalanced datasets ( $\mathrm{He}$ and Garcia, 2009).

\section{Area under the Receiver Operating Characteristics}

AuROC (Hanley and McNeil, 1983) is a score, whose best value is 1 and worst is 0.5 . Receiver Operating Characteristics (ROC) is a plot showing the true positive rate versus false positive rate for a classifier. AuROC is the area under this curve, which is computed using the trapezoidal rule. A perfect classifier has an area of 1 . AuROC actually is an area and hence should have values less than 0.5 as well. However, we can always swap our definitions of what we call 'positive' and 'negative' classes to get an AuROC score of greater than 0.5. We get an AuROC score of 0.5 for a completely random classification.

False Positive Rate FPR is defined as the ratio of false positives to all the class observations that belong to the FALSE class in the dataset. It is defined as,

$$
\frac{F P}{T N+F P} \text {. }
$$

Additionally, since our problem is a binary classification problem, it is also equal to 1 - specificitiy.

\section{PULSAR DATASET and SMOTE}

\subsection{Pulsar Detection}

We briefly outline the steps involved in the detection of pulsars from raw radio data using periodicity searches. More details on each of these steps can be found in Lorimer and Kramer (2004) and Lyon et al. (2016) and references therein. The first major step in the pulsar search pipeline after data digitization, channelization, RFI excision and 'clipping' (Hogden et al., 2012), involves de-dispersion, which corrects for the frequency dependent delay the incoming signals experience due to the presence of free electrons in the interstellar medium. The amount of dispersion is governed by the integrated electron density along the line of sight, also known as the dispersion measure (DM). However, the DM is generally not known in advance. Therefore, we must search over a large range of possible DMs, to find the optimum DM that achieves the highest $\mathrm{S} / \mathrm{N}$ for a suspect detection. A brute force search algorithm for pulsars involves a grid search in DM and acceleration space. For each putative DM and acceleration, periodic signals are searched for using FFT techniques and potential pulsar candidates are then selected from these searches. These candidates are then manually inspected to identify the pulsar-like sources, which later can be analyzed.

\subsection{HTRU Survey}

The dataset we have used in this manuscript for testing various algorithms is obtained through the reprocessing of data from the Southern High Time Resolution mid-latitude survey (Keith et al., 2010) by the PEASOUP pipeline. Briefly, the Southern HTRU Latitude survey (HTRU-S) is a survey of the southern sky $\left(30^{\circ}>l>-120^{\circ} ;|b|<15^{\circ}\right)$, carried out using the 13-beam receiver on the Parkes Radio Telescope to detect radio pulsars and also short-duration radio transients. A similar survey of the Northern skies has also been conducted using the Effelsberg radio telescope with a seven-beam receiver $(\mathrm{Ng}$, 2013). More details of the HTRU-S survey strategy and specifications can be found in Keith et al. (2010). This data has been reprocessed using a GPU-based PEASOUP pipeline with acceleration searches turned on (Morello et al., 2014) (hereafter M14). The pipeline searched for dispersion measures between 0 and $400 \mathrm{~cm}^{-3} \mathrm{pc}$ and accelerations between -50 and $50 \mathrm{~m} / \mathrm{s}^{2}$. M14 manually processed their candidate set and constructed a dataset for machine learning purposes. This dataset consisted of 1200 real pulsars and to this, 90,000 non-pulsar observations (from RFI and other sources of noise) were added. The pulsar candidates encompass a diverse range of periods, duty cycles, and signal to noise ratios. It is unlikely that there are undiscovered pulsars in the set of noise candidates. This dataset (with the pulsar and noise candidates tagged for each event), was made publicly available by M14 for the development and testing of various machine learning algorithms by the wider scientific community. Our goal is to find a machine learning solution with the maximum efficiency in detecting pulsars and minimum contamination from noise candidates. In machine learning parlance as discussed earlier, we are tackling a binary classification problem. 
Each pulsar candidate is described in a PulsarHunter Candidate XML file, having the extension . phcx. See Figure 3.2 to view the schema of the $\mathrm{xml}$ tree.

For all the machine learning algorithms herein, we have used the same feature set as defined by M14. They are listed below, and henceforth we shall denote each feature with a corresponding numerical index (called feature index, $f_{I D}$ ), using 0 -based indexing. More details on each of these features can be found in Lorimer and Kramer (2004). We have also included a Python script file in the Github repository, by the name of ExtractFeatures, which can be used to extract the following features from the PHCX files.

0. S/N Ratio of the folded profile: $\mathrm{S} / \mathrm{N}$ ratio is a measure of the signal significance and is defined for a contiguous pulse window $W$ as (Lorimer and Kramer, 2004; Morello et al., 2014),

$$
S / N=\frac{1}{\sigma \sqrt{w}} \sum_{s_{i} \in W}\left(s_{i}-\bar{b}\right),
$$

where $s_{i}$ is the amplitude of the $i$-th bin of the folded profile, $w$ is the width of the pulse region in each bin, $\bar{b}$ and $\sigma$ represent the mean value and the standard deviation of the folded profile in the off-source region. Because of the large dynamic range of the $\mathrm{S} / \mathrm{N}$ ratio, its logarithm is used as a feature.

1. Intrinsic equivalent duty cycle of the pulse profile: This is an approximate proxy for the total duration of the signal and is defined as the the ratio of the pulse width to its spin period. However, for millisecond pulsars one needs to correct for the large duty cycles due to dispersive smearing. The intrinsic equivalent duty cycle of a candidate is defined as,

$$
D_{e q}=\frac{w_{e q}-\Delta \tau}{P},
$$

where $P$ is the period of the candidate and $\Delta \tau$ is the approximate dispersive smearing time and is defined in M14.

2. Ratio between barycentric period and dispersion measure (log scale): This is the logarithm of the ratio of the period and the dispersion measure.

3. Validity of dispersion measure: This is a figure of merit to bifurcate the dispersion measures of pulsars and RFI signals and is defined as,

$$
V_{D M}=\tanh \left(D M-D M_{\min }\right),
$$

where $D M$ is the dispersion measure of the candidate and $D M_{\min }=2$ for HTRU data. This feature is used to reject non-pulsar candidates below a certain threshold.

4. Persistence of signal in the time domain: This is based on the ansatz that a true pulsar will be consistently visible in the time domain for most of the observation and is defined following Lee et al. 2012,

$$
\chi(s)= \begin{cases}1-\exp (-s / b) & \text { if } s \geq 0 \\ s / b & \text { if } s<0\end{cases}
$$

where $s$ is the $\mathrm{S} / \mathrm{N}$ ratio of a candidate in a subintegration, and $b$ is the benchmark $\mathrm{S} / \mathrm{N}$ ratio, which is a user-defined parameter and the choice adopted by M14 is $b=\frac{2 S_{\min }}{\sqrt{n_{\text {sub }}}}$.

5. RMS distance between the folded profile and the subintegrations. This is a measure of the variability of the pulse shape from the observations. The folded profile as well as every sub-integration is normalized to values between 0 and 1 . The root mean square distance between the folded profile of candidate and each of its subintegrations is defined as,

$$
D_{R M S}=\sqrt{\frac{1}{w n_{s u b}} \sum_{i \in W} \sum_{j}\left(p_{i}-s_{i j}\right)^{2}},
$$

where $p_{i}$ is the value of the $i$-th bin of the folded pulse profile, $s_{i j}$ is the value of $i$-th bin of the $j$-the subintegration.

All of the above features were scaled to have zero mean and unit variance, before training. There are only 1, 192 pulsar observations in the dataset, whereas there are 87,680 non-pulsar observations. For completeness, we report the performance of all the ML algorithms on both the original dataset and on an artificially inflated version of the dataset using Synthetic Minority Over-sampling Technique (SMOTE) (described in the next subsection), which accounts for the mismatch between the ratio of the pulsar to non-pulsar candidates. The motivation behind doing so is to compare how these algorithms perform on highly skewed datasets and also not to bias the results from the ML algorithms.

\subsection{SMOTE}

Synthetic Minority Over-sampling Technique, popularly known as SMOTE (Chawla et al., 2002), is a very well known technique employed in high class imbalance problems. We note that high class imbalance occurs when the class distribution in a classification problem is skewed toward a particular category (either in the training data, or the real-world data). A ratio of 1:10 is generally considered as imbalanced, and 1:100 or greater is considered as highly imbalanced (He and Garcia, 2009). Our dataset has a ratio of 1:73 making it a high class imbalance problem. The crux of this technique involves manufacturing artificial vectors in the feature space to create synthetic datasets, so as to make the ratio more uniform. This generation of mock data is done until the ratio of the minority to the majority class population reaches a user specified threshold, which is passed as an argument to the SMOTE algorithm. For our implementation of SMOTE, k-Nearest Neighbors (kNN, Altman 1992, Chapter 13 of Hastie et al. 2001) is employed in the process of creation of synthetic feature vectors. For every feature 


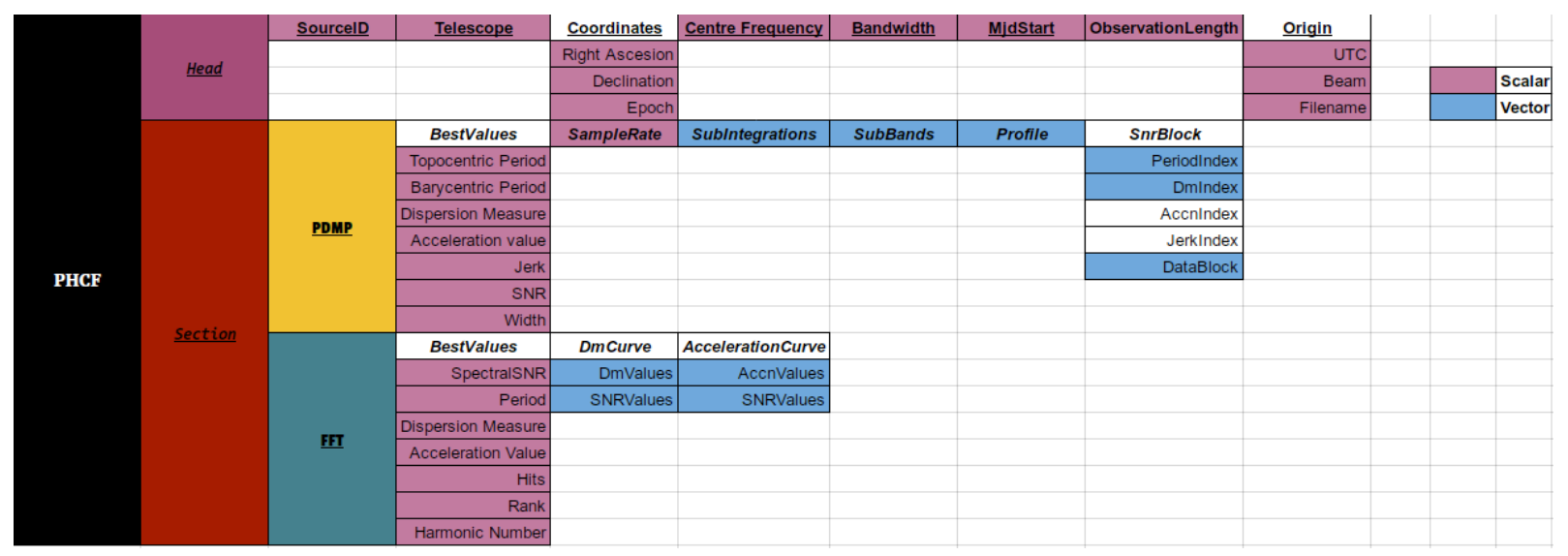

Figure 1: Schema of the XML tree containing details of the pulsar dataset from the HTRU survey, described in detail in M14 and made publicly available. Each candidate file has the above schema and structure.

vector belonging to the minority class, it's $k$ nearest neighbors are determined, from which a random neighbor is chosen and in-between this randomly chosen neighbor and initial feature vector, synthetic feature vectors are interpolated randomly. This is a bird's-eye view of the algorithm. Interested readers should refer to Chawla et al. (2002) for more details.

SMOTE has been previously used for the classification of unknown point sources from the Fermi-LAT catalog (Abdo et al., 2013) and classification of variable stars from Kepler (Bass and Borne, 2016). Two years ago, it has also been used in a pulsar search pipeline to address the class imbalance problems (Devine et al., 2016).

We run all our machine-learning algorithms on two datasets, the original one, on which no SMOTE has been applied, which we refer to as "non-SMOTE" applied data, and another one with SMOTE applied, which we refer to as "SMOTE". We use SMOTE to artificially balance the dataset, i.e. to have a 1:1 ratio of pulsar to non-pulsar samples.

Our approach of addressing the high class imbalance problem is complementary to what M14 have done. M14 performed oversampling of the pulsar-only dataset, until they have four non-pulsar (negative) samples for each pulsar (positive) sample in the training dataset. On the contrary, we employed SMOTE to artificially balance the dataset. We have used the Python module called imbalanced-learn to apply the SMOTE technique to our datasets.

\section{MACHINE LEARNING METHODS}

In this work, we have applied four different algorithms to this pulsar classification problem:

\section{Artificial Neural Network (ANN)}

2. Adaboost

3. Gradient Boosting Classifier (GBC)

4. eXtreme Gradient Boosting (XGBoost)
To the best of our knowledge, among these four algorithms, Adaboost, GBC, and XGBoost have never been used before in the core pulsar search pipelines or during the post-processing of pulsar candidates. Although, we are only reporting the aforementioned techniques, we also tried the Naïve Bayes algorithm. However, the performance of the Naïve Bayes was unsatisfactory. The core premise of the Naïve Bayes algorithm is conditional independence between the features for a given target label. We find that there is significant amount of correlation between the features. Hence, Naïve Bayes fails on this dataset (Zhang, 2004). Therefore, we do not report the results from the Naiive Bayes algorithm. We note that this agrees with the conclusions from Devine et al. (2016) and Smithbauer (2013), who also found that Naïve Bayes performs poorly on pulsar data. However, Lyon et al. (2014) and Lyon et al. (2016) observed reasonable performance with this algorithm using a different feature set on the HTRU-1, HTRU-2, and LOTAAS-1 datasets.

We now describe in detail our implementation of the four algorithms and present their classification results. All four algorithms have been implemented in Python using the scikit-learn module (Pedregosa et al., 2011). The implementation code, along with the dataset, is available in the Github repository ${ }^{3}$. We have also added the Python code to generate the dataset (CSv) file from the PHCX Candidates files. More details about the code can be found in the Github repository. In a typical $k$-fold cross-validation $(\mathrm{CV})$ procedure, the entire dataset is split into $k$-folds and the classifier under test is trained on $(k-1)$-folds and assessed on the remaining one fold, which was not used for training. This process is repeated $k$ times so that the classifier under test can be assessed (by computing the scores) on each fold of the dataset. To further reduce the variance in the scores, the complete $k$-fold $\mathrm{CV}$ is performed multiple times and the scores are averaged over these iterations. A variant of the $\mathrm{CV}$ procedure, where the dataset is judiciously broken into folds such that the ratio of positives to negatives in each fold is preserved as best as possible, is called stratified

\footnotetext{
${ }^{3}$ https://github.com/shiningsurya/pulsarml
} 
Table 2: Hyper parameters of all the machine learning (ML) models discussed here. We have used the same set of hyper parameters for both the non-SMOTE and SMOTE datasets.

\begin{tabular}{|c|c|}
\hline $\mathrm{ML}$ & Hyper-parameters \\
\hline ANN(MLP) & $\begin{array}{l}6: 6: 4 \text { network } \\
\text { activation function : Leaky RLeU } \\
\text { output layer : softmax } \\
\text { learning rule : adam } \\
\text { learning rate }=0.001 \\
\text { batch size }=32 \\
\text { number of iterations }=750\end{array}$ \\
\hline Adaboost & $\begin{array}{l}\text { number of estimators }=250 \\
\text { learning rate }=0.1 \\
\text { base estimator: decision stump }\end{array}$ \\
\hline $\mathrm{GBC}$ & $\begin{array}{l}\text { number of estimators }=250 \\
\text { learning rate }=0.1 \\
\text { max depth }=5 \\
\text { minimum number of samples at leaf node }=2\end{array}$ \\
\hline XGBoost & $\begin{array}{l}\text { number of estimators }=500 \\
\text { learning rate }=0.001 \\
\text { maximum depth }=3 \\
\text { regularization alpha }=0.0001 \\
\text { gamma }=0.1\end{array}$ \\
\hline
\end{tabular}

$\mathrm{CV}$, which we use here. In the non-SMOTE case, the classifier under test is simply trained on the training fold. However, in the case of SMOTE, we employ SMOTE in the CV procedure to artificially balance the training fold (and only the training fold) and on this inflated training fold, the classifier under test is trained. It is crucial to note that in both the cases (SMOTE and non-SMOTE), the classifier is tested on the same test fold. We also note that the hyper parameters for all the ML models (cf. Table 2) are the same in both the non-SMOTE and the SMOTE cases.

We carry out 20 iterations of 5-fold stratified CV for each of the machine learning algorithms discussed in this work using the procedure discussed above. We therefore get 100 values for each of the scores, which we aggregate and report.

\section{1. $A N N(M L P)$}

An Artificial Neural Network (ANN) is a popular tool in the machine learning community. Complete details of an ANN can be found in Bishop (2006) and Hastie et al. (2001). Most machine learning algorithms used by the pulsar community are some variant of the ANN. ANNs are also widely used in optical astronomy (Sadeh et al., 2016), in particular for photometric redshift estimation (eg. Desai et al. 2012).

We have used a simple Multi-Layer Perceptron (MLP) similar to M14. The hyper parameters used for training the ANN are specified in Table 2. We make use of the SKNN Python module to implement this MLP. ${ }^{4}$

\footnotetext{
${ }^{4}$ Please note of the dependency issue of SKNN with theano.
}

More details on our implementation can be found in the Github repository. The hyper parameters, along with all the connection weights, form a functional mapping from the input features to the output (target variable). The loss function is defined on the predicted target and the true target (which comes from the labeled data).

Minimization of this loss function is a high dimensional non-convex problem. Hence, we use a gradient-based method to perform the optimization. The heuristics are as follows: Our choice of hyper parameters and training data yields a specific level of training error. We then compute the gradient and update the connection weights accordingly (in the direction of negative gradient) so as to reduce the training error. Our aim is to find the most optimum set of connection weights that lead to the lowest training error. The most crucial step here is the computation of the connection weights from the gradient, and for this purpose, we employ the learning rate. Learning rate is defined as the hyper parameter which regulates the magnitude of the distance we want to traverse against the direction of its gradient. For all the gradient-based solutions, determining the optimal learning rate is often the trickiest problem. If we choose too high a value of this hyper parameter, we might never converge to the global minima (maxima) but could pass right by it, whereas too low a value of the learning rate could possibly increase the convergence time and the possibility of getting stuck in a local minimum (maximum). At times, finding an optimal parameter becomes so difficult that switching to alternate machine learning solutions may seem more prudent. In order to mitigate this issue, we have used Adam (a method for stochastic optimization) as our gradient descent optimization algorithm (learning rule), instead of the default Stochastic Gradient Descent (SGD) in SKNN. In the SGD optimization algorithm, the learning rate is either a constant or is reduced after a fixed number of epochs. Adam, instead computes the running first and second-order (un-centered) moments of the gradients and dynamically adjusts the learning rate on the fly. The base learning rate ('original learning rate') is normalized by dividing by the square-root of the non-centered and bias corrected second-order moment of the gradients, and followed by scaling using the running bias-corrected first-order moment of the gradient as the scale parameter. It is important to note that these gradients essentially decay exponentially, since the neural network is converging. There is also an additional fudge factor added to this square-root (second order moment), in order to ensure that the division operation doesn't throw a 'divide-by-zero' exception. More details about Adam can be found in Kingma and $\mathrm{Ba}$ (2014). It is also worthwhile to note that Adam draws it's name from "ADAPTIVE" moments, since the running moments determine the learning rate.

We note that M14 employed a single hidden layer with eight hidden units and used tanh as the output layer activation function. They have trained using the 'mini-batch' approach, wherein the weight updates happen after a batch (subset) of the data has been processed. On the other hand, we have implemented a 6:6:4 MLP and also used a different learning rule (Adam), activation function (Leaky RLeU Maas et al. 2013), and the output layer of softmax (Hinton and Salakhutdinov, 
2009). The softmax layer ensures that the final output lies between $[0,1]$ (analogous to probabilities), contrary to tanh function, whose range is $[-1,1]$.

\subsection{Adaboost}

Adaboost, an acronym for Adaptive Boosting, takes a collection (an ensemble) of 'weak learners' and recursively trains them on copies of the original dataset, all the while focusing on the 'difficult' (or outlier) data points (hence the word 'Adaptive') (Freund and Schapire, 1995). This, in turn, ensures that such an ensemble performs drastically better on the test data. A natural choice of weak learners are simple rules or logic, which are realized as decision trees, also known as algorithmic flowcharts (Breiman et al., 1984).

Those decision trees which are of depth one, are called 'decision stumps' (Ai and Langley, 1992). More details about Adaboost can be found in Section 2 of Mayr et al. (2014). Adaboost has been shown to outperform other machine learning algorithms for many different supervised classification problems in optical astronomy (Hoyle et al., 2015; Sevilla-Noarbe and Etayo-Sotos, 2015; Elorrieta et al., 2016; Acquaviva, 2016; Zitlau et al., 2016).

We have used the same implementation of Adaboost as in Hoyle et al. (2015), where it was applied to photometric redshift estimation of SDSS DR10 data and shown to be more robust against outliers and provided better matches with spectroscopic redshifts than the ANNs. We have defined our Adaboost model using two properties (specified in Table 2), viz., the number of trees and the learning rate. The former corresponds to the number of weak learners in the ensemble and the latter determines how strongly each weak learner should contribute to the weights. For a large number of estimators, each estimator would have it's own effect on calculating the weights, which would get enhanced strongly. On the contrary, for a smaller number of estimators, the weights assigned may not be sufficient for the model to learn a 'tricky' sample (hard to classify). In other words, there exists a trade-off between the number of estimators and the learning rate. Please note that Adaboost is a meta-classifier (Sammut and Webb, 2011), which takes $\mathrm{N}$ copies of the weak learner and trains them on the same feature set but with different weights assigned. These $\mathrm{N}$ copies correspond to the number of weak-learners. See Belanich and Ortiz (2012), which extols the power of Adaboost on mathematical grounds.

\subsection{Gradient Boosting Classifier}

A Gradient Boosting Classifier (GBC) is a form of ensemble classification system. Much like Adaboost, which was discussed in the previous subsection, GBC iteratively adds simple classifiers (decision trees, although not necessarily of depth one), which are successively trained on the errors of the predecessor classifier. In other words, the very first classifier of the ensemble is trained on the dataset, whereas the second classifier is trained on the errors of the first classifier and added to the first classifier and so on. The merging takes place in an aggressive fashion; the successor tree couples with the predecessor tree using a coupling parameter, which is optimized so that the error of the combined system is minimized. Note that, this is formally a linear optimization problem. This hierarchical procedure is also known as boosting. See Friedman (2001); Tramacere et al. (2016) for a thorough understanding of Gradient Boosting Machines. Gradient boosting has also been widely used in astrophysics for photometric classification of Type 1a supernovae (Möller et al., 2016) and for automated galaxy detection and classification using the Galaxy Zoo catalog (Tramacere et al., 2016). Another hyper parameter in GBC is the 'learning rate', which governs how strongly a classifier should be merged with it's predecessor. Learning rate lends itself to GBC as a regularization mechanism. In GBC, we employ 4 hyper parameters: learning rate as defined above, number of estimators (n_estimators, number of trees in ensemble), maximum depth, and minimum samples per leaf, the last two are actually hyper parameters of the decision tree (member of the ensemble). We note that all the members of the ensemble have the same hyper parameter set which can be found in Table 2 . We followed the same procedure as discussed at the start of this section. We used the scikit-learn implementation of GBC.

\subsection{XGBoost}

XGBoost (Chen and Guestrin, 2016), is an acronym for eXtreme Gradient Boosting, and works in the same way as Gradient Boosting but with the addition of Adaboost-like feature of assigning weights to each sample.

XGBoost is a tree-based model which gained a lot of popularity right since it's inception in the machine learning community in 2016. This model was also the winner of HEP meets $M L$ Kaggle challenge (Chen and He, 2015) and our problem is no exception to that. In astrophysics, XGBoost was recently used for the classification of unknown point sources in the FermiLAT catalog (Mirabal et al., 2016) and also for studying the stability of planetary systems using physically motivated features with the help of supervised learning (Tamayo et al., 2016).

Unlike in GBC, where every member of the ensemble (tree) is built sequentially, XGBoost on the other hand, can parallelize this task and give substantial speed boost (See Sections 4,5 of Chen and Guestrin (2016) and references therein). Moreover, the regularization techniques, such as L1 and L2, which are used to control over-fitting are available in XGBoost, but not in Adaboost and GBC. Another key feature of XGBoost is the scalability it offers (Alves, 2017). This means that it can be efficiently run on distributed systems and can also work on very large datasets with ease ${ }^{5}$. XGBoost can also be trained using Graphical Processing Units (GPUs, See Mitchell and Frank 2017) and can offer extremely high speed boosts. While our dataset is not as onerous as those on which XGBoost is usually run, ours is the first proof of principles application of XGBoost to the pulsar classification problem.

XGBoost is readily available as a Python package, which is used in this work. We use five hyper parameters for training in

${ }^{5}$ Refer to this url for the list of use-cases: https://github.com/ $\mathrm{dmlc} / \mathrm{xgboost} / \mathrm{blob} / \mathrm{master} /$ demo/README. md\#usecases 
XGBoost. We build an ensemble of 500 estimators with a learning rate of $0.1 \%$ (doubled the number of trees compared with Adaboost, GBC because learning rate was changed to 0.001 to account for the 'learning-rate/number of trees ${ }^{6}$ trade-off ${ }^{6}$ ). Every tree in the ensemble has a maximum depth of 3 with $L 1$ regularization parameter of $0.01 \%$. We have also used the gamma hyper parameter which controls the tree building process by constraining the amount of gain a split should have.

\section{Feature Selection}

We now delve into the process of feature selection. In any supervised ML algorithm, the features that a model learns from largely determine the accuracy of the model. An informationtheoretic way to quantify the efficacy of a feature is to compute the Mutual Information (MI) between a feature and its corresponding class label (Macedo et al., 2017). MI is defined as the amount of information conveyed by one random variable through another random variable (Here, we treat the features as some random variables). Mathematically,

$$
M I=\iint p(x, y) \log \frac{p(x, y)}{p(x) p(y)},
$$

where $p$ represents the probability density function (p.d.f), whereas $x$ and $y$ are two continuous random variables. Estimating the entropy from first principles (by computing the p.d.f from the histogram and carrying out the integration) is a very computationally challenging task. To alleviate this, we use the sklearn. Featureselection ${ }^{7}$ module, which has functions to compute MI. These functions actually use the estimator which is based on Kraskov et al. 2004.

We use the minimum Redundancy Maximum Relevance (mRMR) procedure (Peng et al., 2005; He et al., 2013; Macedo et al., 2017) to determine the feature rankings using the nonSMOTE and SMOTE datasets. This procedure is widely used in the machine learning community for feature selection (Chandrashekar and Sahin, 2014), as it provides an algorithmagnostic criterion for selecting the best feature and its computational cost is not onerous. Although, mRMR has never been used in electromagnetic astronomy before (to the best of our knowledge), it is routinely used in neutrino astronomy, in particular for the IceCube event selection pipeline to select the neutrino events from the background of cosmic ray muons (The IceCube Collaboration et al., 2015). For this procedure, we assign a rank to the feature based on it's score which is defined as:

$$
\text { Score }_{i}=\operatorname{MI}\left(x_{i} ; y\right)-\frac{1}{d-1} \sum_{j \neq i} \operatorname{MI}\left(x_{i} ; x_{j}\right),
$$

where $x_{i}$ denotes the $i^{\text {th }}$ feature column and $d$ represents the number of features. This score takes into account the mutual

\footnotetext{
${ }^{6}$ For a large number of trees, higher learning rate would make our model over-fit to the data. Hence, a smaller learning rate should go with a larger number of trees so that our model can learn the overall structure and not the intricate nuances (noise) of the signal

${ }^{7}$ This functionality was added in version 0.19
}

information (MI) between a feature and its class label (or how powerful the feature is) and also penalizes for any correlations between the features. Naturally, higher the score, more conspicuous is the feature, and hence its rank should be higher. MI between a feature and the corresponding class label (relevance) doesn't involve any other feature, and hence does not measure the inter-feature relationships. Therefore, by definition, mutual information does not include any correlation between the features. The score is defined in such a way to account for 'relevance' and 'redundancy'.

Tree-based ML methods, discussed in this work, provide 'feature importances' out of the box. At the grassroot level of any tree-based ML method, a feature is chosen, which is used to make a split (corresponding to a branch in the decision tree), and the number of times a feature is chosen to split can be treated as a measure of the importance of the feature. However, in practice the maximum reduction in the error when a feature is used to make the split is used to gauge it's feature importance. But, still our understanding is valid because if a feature is frequently used, it has better descriptive power and naturally is assigned a higher rank. The criteria for selecting a feature to partition the data is related to our problem statement. In a regression setting, the objective is to reduce the variance in the partitions so that every sample in any partition is representative of each other. On the other hand, in a classification setting (such as the problem at hand), only a feature which separates the data in such a way that each partition is homogeneous and doesn't contain any contamination is chosen. However, the motivation that the samples belonging to a particular partition are representative of each other remains the same as in a regression setting. Naturally, features which are chosen larger number of times are more powerful (in terms of their separation ability) and as a natural outcome, the tree-based methods provide additional diagnostic insights about the data on which they are trained. More details on these aspects of feature selection can be found in Hoyle et al. (2015) and Chapter 10 of Hastie et al. (2001).

To interpret the feature rankings coherently, we have presented the data using a stacked bar plot in Fig. 2. For both the non-SMOTE and SMOTE cases, we have four different rankings based on Adaboost, GBC, XGBoost and mRMR. For every rank, we plot the number of times a given feature was assigned that rank in a stacked bar plot, with each feature having a unique color code. For instance, $f_{I D}=0$ ranks in first and second places twice in both the non-SMOTE and SMOTE cases, which means, that this feature has the most descriptive power. This result is in agreement with Lyon et al. (2016). Lastly, we note that the distribution of features with respect to the ranks is similar in both the non-SMOTE and SMOTE cases, which implies that our technique of artificially inflating the dataset has not significantly altered the descriptive power of the features in the dataset.

\section{Results}

We now report the salient results from applications of all the four ML methods. 
Figure 2: Feature rankings as found by Adaboost, GBC, XGBoost, and mRMR approach in this work. We find that the $\mathrm{S} / \mathrm{N}$ Ratio of the folded profile $\left(f_{I D}=0\right)$ to be the most descriptive feature. $f_{I D}=1, f_{I D}=2, f_{I D}=3, f_{I D}=4$ and $f_{I D}=5$, correspond to the Intrinsic equivalent duty cycle of the pulse profile, ratio of barycentric period and DM, validity of DM, RMS distance between folded profile, persistence of signal in the time domain, and the sub-integrations respectively. Refer to last paragraph of Sect. 5 for more details about this plot.
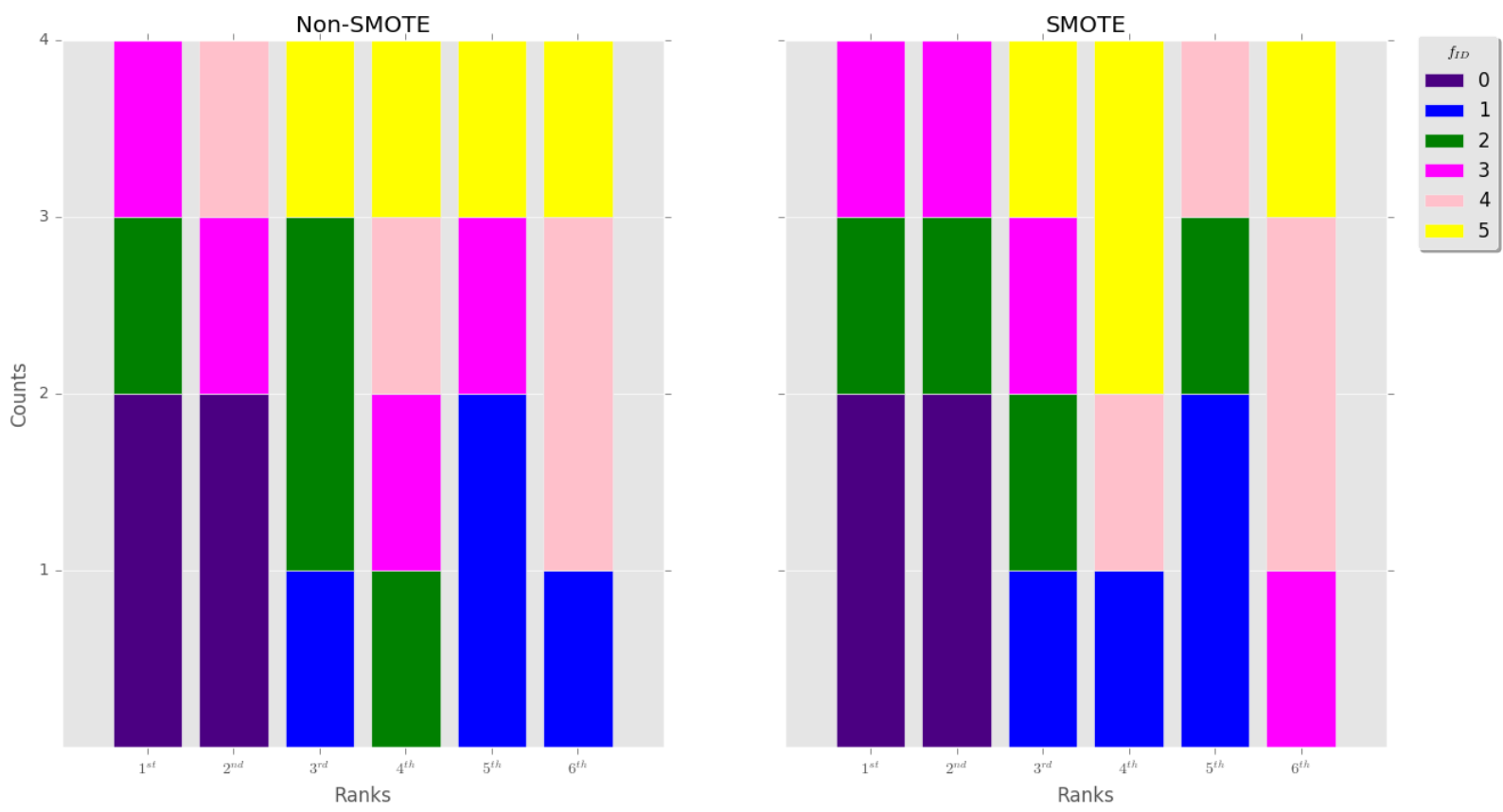

Table 3: Averaged confusion matrices of all the algorithms used in this paper. Elements of the confusion matrix are counts, which are integers. However, we have averaged over the $20 \times 5$-fold Stratified CV, and hence, the floating point numbers are reported for each of the scores. Refer to Section 2 for details about the various definitions used here. The error bars for all the elements of all the averaged confusion matrices are of $O(0.01)$ and hence not reported in the table explicitly. We note that T, F stand for True and False respectively, whereas N, P stand for negative and positive respectively. Refer to Table 1 to understand the confusion matrix. The shaded values are for the SMOTE dataset, whereas non-shaded values are for the non-SMOTE ones.

\begin{tabular}{ccccccccc}
\hline ML Model & \multicolumn{2}{c}{ ANN(MLP) } & \multicolumn{2}{c}{ Adaboost } & \multicolumn{2}{c}{ GBC } & \multicolumn{2}{c}{ XGBoost } \\
\hline & $\mathrm{N}$ & $\mathrm{P}$ & $\mathrm{N}$ & $\mathrm{P}$ & $\mathrm{N}$ & $\mathrm{P}$ & $\mathrm{N}$ & $\mathrm{P}$ \\
$\mathrm{F}$ & 1.4 & 10.4 & 1.2 & 3.3 & 1.1 & 2.9 & 0.8 & 5.2 \\
$\mathrm{~T}$ & 17525.6 & 237.0 & 17532.8 & 237.2 & 17533.0 & 237.3 & 17530.8 & 237.7 \\
$\mathrm{~F}$ & 0.4 & 9.6 & 0.5 & 5.6 & 1.3 & 3.5 & 0.6 & 6.3 \\
$\mathrm{~T}$ & 17526.4 & 238.0 & 17530.4 & 237.9 & 17532.5 & 237.0 & 17529.7 & 237.8 \\
\hline
\end{tabular}

Table 4: Various scores averaged over $20 \times 5$-fold stratified CV. Refer to Section 2 for definitions of the scores used here, and for the methodology applied to compute these scores. Error bars are negligible (variation in the third decimal digit only) for all the scores, and hence not reported here. The shaded values are for the SMOTE dataset, whereas the non-shaded values are for the non-SMOTE ones.

\begin{tabular}{lcccccccc}
\hline ML Model & Recall & Precision & Accuracy & $f_{1}$ & log-loss & G-Mean & AuROC & FPR[\%] \\
\hline ANN (MLP) & 0.990 & 0.964 & 0.999 & 0.977 & 0.021 & 0.995 & 0.994 & 0.059 \\
ANN (MLP) & 0.998 & 0.961 & 0.999 & 0.979 & 0.019 & 0.998 & 0.994 & 0.055 \\
Adaboost & 0.995 & 0.986 & 0.999 & 0.990 & 0.008 & 0.997 & 0.994 & 0.019 \\
Adaboost & 0.997 & 0.976 & 0.999 & 0.986 & 0.012 & 0.998 & 0.994 & 0.032 \\
GBC & 0.995 & 0.987 & 0.999 & 0.991 & 0.008 & 0.997 & 0.994 & 0.017 \\
GBC & 0.995 & 0.986 & 0.999 & 0.990 & 0.008 & 0.997 & 0.993 & 0.020 \\
XGBoost & 0.996 & 0.978 & 0.999 & 0.987 & 0.012 & 0.998 & 0.994 & 0.030 \\
XGBoost & 0.997 & 0.974 & 0.999 & 0.985 & 0.013 & 0.998 & 0.994 & 0.036 \\
\hline
\end{tabular}


The confusion matrices of all the ML algorithms, on both the SMOTE and non-SMOTE datasets are shown in Table 3. A tabular summary of the various quality metrics can be found in Table 4. We make the following observations:

- All the algorithms give approximately the same values for the confusion matrices for both the datasets. The number of false pulsars is largest for the ANN (MLP).

- The recall, accuracy, $f_{1}$ score, G-mean, and AuROC perform about the same for all the four algorithms, for both the SMOTE and non-SMOTE datasets.

- Precision varies across the datasets, depending on the ML algorithm applied. In case of Adaboost, the difference between non-SMOTE and SMOTE precision is the largest, and is smallest in the case of GBC, which is surprising, since both these ML algorithms are tree-based.

- The log-loss for the ANN (MLP) using the non-SMOTE datasets is about an order of magnitude worse than that for the other algorithms. However, they are comparable, when considering the SMOTE datasets.

- For all the algorithms, we have been able to achieve a recall value of close to $100 \%$, but with a FPR about an order of magnitude lower than the value of $0.64 \%$, obtained in M14. Among the four algorithms, ANN (MLP) shows the largest FPRs for both the non-SMOTE and SMOTE cases, compared to the others.

\section{Discussions and Conclusions}

We have run multiple supervised machine learning algorithms to evaluate the efficacy and robustness of the separation of true pulsar signals from noise and RFI candidates, using data from the HTRU-S survey, a part of which has been made publicly available by M14. We have reported our implementation and results from four of these algorithms, namely an ANN Multi Layer Perceptron (ANN (MLP)), Adaboost, Gradient Boosting Classifier (GBC), and XGBoost, all of which report high accuracy and G-mean. The last three algorithms have never been previously applied either in the core pulsar searches, or in any post-processing pipeline used to separate the real pulsars from noise candidates.

In addition to the feature ranking based on importances, as returned by the tree-based machine learning methods (Adaboost, GBC and XGBoost), we have also implemented feature selection using the Minimum Redundancy and Maximum Relevance Feature Selection (mRMR) approach. This is the first application of mRMR in electromagnetic astronomy. To alleviate the high class imbalance (high non-pulsars candidates to pulsars candidates), we have used the Synthetic Minority Oversampling Technique, also known as SMOTE, to artificially balance the datasets. For the same set of hyper parameters, each ML method was trained on the original (non-SMOTE) as well as the SMOTE applied dataset in a $20 \times 5$-fold Stratified CV fashion. We evaluated the various scores for all four of these algorithms and reported the average scores, which can be found in Table 4.

Compared to M14, which achieved a FPR of $0.64 \%$ at a recall of $100 \%$, our ANN (MLP) implementation achieved FPRs of $0.059 \%, 0.055 \%$ for the values of recall of $0.990,0.998$ in non-SMOTE and SMOTE cases respectively, . However, M14 performed over-sampling to alleviate the high-class imbalance, whereas, we did not address it for the non-SMOTE case, and applied SMOTE to balance the dataset in the other case artificially. Our alternate implementation of ANN (MLP) as well as the tree-based ML methods resulted in FPRs about an order of magnitude lower than that of M14 for both the non-SMOTE and SMOTE cases.

An ideal classifier must be able to classify both the pulsars and non-pulsars correctly. In other words, it should have zero misidentified pulsars and zero misidentified non-pulsar candidates. This essence is conveyed in the accuracy, but, given the high-class imbalance in the dataset, the accuracy score is susceptible to be misleading. An 'all-negative' classifier (which labels all the candidates as a non-pulsar irrespective of input data) would report an accuracy of 0.9 , if applied to a dataset with 10 pulsars (positive instances) and 90 non-pulsars (negative instances), whereas recall, precision, $f_{1}$, G-Mean would all vanish. To alleviate this issue, we have employed SMOTE to artificially inflate the pulsar-set (positive instances) to make the dataset more balanced. We note that the SMOTE dataset led to no significant improvement in the scores compared to the non-SMOTE dataset, which is counterintuitive.

One possible reason for the above result could be that the tree-based algorithms are inherently more robust to class imbalance. Similar results have also been observed by Lyon et al. (2014, 2016), wherein Gaussian Hellinger Very Fast Decision Tree (GH-VFDT) was shown to maximize the classification performance in heavily imbalanced data streams. In the case of an ANN (MLP), where the class imbalance is known to have a strong impact on its performance, we assert that our choice of learning rule and amount of iterations for which we need to train, allowed the ANN (MLP) to overcome the high classimbalance at the expense of large amount of training time and computational resources.

Tree-based machine learning algorithms automatically yield 'feature importances', which provide many insights about the data. We have reported these feature importances along with $\mathrm{mRMR}$ based rankings on both the non-SMOTE and the SMOTE datasets (cf. Fig. 2.) Considering all the feature rankings, we found that the $\mathrm{S} / \mathrm{N}$ of the folded profile $\left(f_{I D}=0\right)$ was the most descriptive feature. Note, that this is in agreement with similar conclusions from Lyon et al. (2016).

In our set of machine learning methods, which we have successfully implemented, we note that recall, accuracy, and GMean varied only after the third significant digit. Therefore, for our dataset it was not possible to comparatively rank these algorithms on the basis of the above performance scores. So, we considered a heuristic approach, based on qualitative aspects to compare them. ANNs, which have been shown to work quite well on classification tasks such as this in earlier works provide no diagnostic information or interpretation of what is happen- 
ing 'behind the scenes'. Interpretation of ANNs is a long standing problem in the Machine learning community, which renders ANNs as nothing more than black boxes (See (Benitez et al., 1997; Mantas et al., 2006), which try to provide some insight into the working of an ANN). Furthermore, they require huge amount of computational resources for training and testing, and offer a vast palette of hyper parameters, which makes it somewhat cumbersome to tune. While there are still some use-cases where, the ANNs and their variants, e.g. Convolutional Neural Networks (CNNs), are the state-of-the-art and unmatched by any other machine learning methods, the problem at hand is not one, where the choice of an ANN would be ideal. Tree-based methods, on the other hand, seem very well suited as they are fast in training/testing, provide powerful insights about the data and are much simpler to train.

Adaboost, which is strictly not a tree-based ML method but a meta-classifier also does well on the problem at hand. It assigns and manipulates the weights of each datum in the training set so that even the 'tricky' points are modeled and accounted for. One immediate concern this methodology raises is: how would it perform on noisy data? The scores reported may obfuscate the underlying requirement of clean, superior quality data for the algorithm to train on, which unfortunately, is never at our disposal all the time.

The ideology of Gradient Boosting Classifier (GBC) to sequentially train the classification trees on the errors of the predecessor tree in an additive fashion is almost identical to that of Adaboost. The main high level difference between the two algorithms is that, Adaboost assigns a high weight to the 'tricky' datum, whereas GBC takes the help of another tree to learn that 'tricky' datum. Quite interestingly, the idea of assigning weights was conceived before the idea of using the gradient, which is used to train the successor trees. Adaboost were first used in 1995 and GBC in 2001. Similar to the ANNs, the learning rate hyper parameter plays a decisive role in controlling the performance of GBC. One serious drawback of GBC is its sequential algorithm, which makes it powerless in the face of an enormous onslaught of data.

We note that XGBoost supersedes Adaboost and GBC, and provides a parallelization capability (including GPU support), has the ability of regularization, and is tailor-made for extreme value problems. In the SKA era, which is akin to Big Data era for the radio astronomers, one would expect XGBoost to be the de-facto algorithm for most ML purposes.

We end, by pointing out that our results and conclusions are based on a single dataset and feature set, used in Morello et al. (2014), and we have not yet tested these algorithms on other datasets. So it is possible that our results are specific to the dataset been used. We do plan to implement these algorithms on other publicly available pulsar datasets (for eg. Lyon et al. 2016) in future works. We also plan to apply other ML-based post-processing pipelines such as PICS (Zhu et al., 2014) on this same dataset.

We hope that our results based on publicly available postprocessing data from the HTRU-S survey processed with the PEASOUP pipeline provide impetus to the pulsar community to consider implementing XGBoost as well as Adaboost and GBC in their pulsar (and other radio transient search) pipelines to aid in the automated separation of RFI and other noise candidates. Conversely, we hope that this work spurs other pulsar groups to make their post-processing data public (similar to what is done for optical large-scale structure photometric and spectroscopic surveys), so that people outside the pulsar community can run and tune their favorite machine learning algorithms on these datasets. We have also made available our ipython notebooks containing all the codes to reproduce our results at https: //github.com/shiningsurya/pulsarml

\section{Acknowledgments}

We would like to thank Robert Lyon and Vincent Morello for invaluable feedback and comments on the manuscript, and to Vincent Morello and Ewan Barr for useful correspondence about the HTRU dataset and the SPINN algorithm, and for making their data public. We are also grateful to P.K. Srijith and Ben Hoyle for many enlightening discussions about machine learning.

\section{References}

Abdo, A.A., Ajello, M., Allafort, A., Baldini, L., Ballet, J., Barbiellini, G., Baring, M.G., Bastieri, D., Belfiore, A., Bellazzini, R., et al., 2013. The Second Fermi Large Area Telescope Catalog of Gamma-Ray Pulsars. ApJS 208, 17. 1305.4385

Acquaviva, V., 2016. How to measure metallicity from five-band photometry with supervised machine learning algorithms. MNRAS 456, 1618-1626. 1510.08076.

Ai, W.I., Langley, P., 1992. Induction of one-level decision trees, in: Proceedings of the Ninth International Conference on Machine Learning, Morgan Kaufmann. pp. 233-240.

Altman, N.S., 1992. An introduction to kernel and nearest-neighbor nonparametric regression. The American Statistician 46, 175185. http://www.tandfonline.com/doi/pdf/10.1080/ 00031305.1992 .10475879

Alves, A., 2017. Stacking machine learning classifiers to identify Higgs bosons at the LHC. Journal of Instrumentation 12, T05005. 1612.07725.

Baghram, S., Afshordi, N., Zurek, K., 2011. Prospects for detecting dark matter halo substructure with pulsar timing. Phys. Rev. D 84, 043511. 1101. 5487.

Ball, N.M., Brunner, R.J., 2010. Data Mining and Machine Learning in Astronomy. International Journal of Modern Physics D 19, 1049-1106. 0906.2173.

Bass, G., Borne, K., 2016. Supervised ensemble classification of Kepler variable stars. MNRAS 459, 3721-3737. 1604.01355.

Bates, S.D., Bailes, M., Barsdell, B.R., Bhat, N.D.R., Burgay, M., BurkeSpolaor, S., Champion, D.J., Coster, P., D’Amico, N., Jameson, A., Johnston, S., Keith, M.J., Kramer, M., Levin, L., Lyne, A., Milia, S., Ng, C., Nietner, C., Possenti, A., Stappers, B., Thornton, D., van Straten, W., 2012. The High Time Resolution Universe Pulsar Survey - VI. An artificial neural network and timing of 75 pulsars. MNRAS 427, 1052-1065. 1209.0793.

Belanich, J., Ortiz, L.E., 2012. On the Convergence Properties of Optimal AdaBoost. ArXiv e-prints 1212.1108.

Benitez, J.M., Castro, J.L., Requena, I., 1997. Are artificial neural networks black boxes? IEEE Transactions on Neural Networks 8, 1156-1164.

Bishop, C.M., 2006. Pattern Recognition and Machine Learning (Information Science and Statistics). Springer-Verlag New York, Inc., Secaucus, NJ, USA.

Blandford, R.D., 1992. Pulsars and Physics. Philosophical Transactions of the Royal Society of London Series A 341, 177-192.

Breiman, L., 2001. Random forests. Machine learning 45, 5-32.

Breiman, L., Friedman, J.H., Olshen, R.A., Stone, C.J., 1984. Classification and Regression Trees. Wadsworth and Brooks, Monterey, CA. 
Cameron, A.D., Barr, E.D., Champion, D.J., Kramer, M., Zhu, W.W., 2017. An investigation of pulsar searching techniques with the fast folding algorithm. MNRAS 468, 1994-2010. 1703.05581.

Cavuoti, S., 2013. Data-rich astronomy: mining synoptic sky surveys. ArXiv e-prints 1304.6615.

Chandrashekar, G., Sahin, F., 2014. A survey on feature selection methods. Computers \& Electrical Engineering 40, 16-28.

Chawla, N.V., Bowyer, K.W., Hall, L.O., Kegelmeyer, W.P., 2002. Smote: Synthetic minority over-sampling technique. J. Artif. Int. Res. 16, 321-357.

Chen, T., Guestrin, C., 2016. XGBoost: A Scalable Tree Boosting System. ArXiv e-prints 1603.02754.

Chen, T., He, T., 2015. Higgs Boson Discovery with Boosted Trees, in: Cowan, G., Germain, C., Guyon, I., KÃl'gl, B., Rousseau, D. (Eds.), Proceedings of the NIPS 2014 Workshop on High-energy Physics and Machine Learning, PMLR, Montreal, Canada. pp. 69-80.

Cohen, W.W., 1995. Fast effective rule induction, in: Proceedings of the twelfth international conference on machine learning, pp. 115-123.

Cortes, C., Vapnik, V., 1995. Support vector machine. Machine learning 20, 273-297.

Desai, S., Armstrong, R., Mohr, J.J., Semler, D.R., Liu, J., Bertin, E., Allam, S.S., Barkhouse, W.A., Bazin, G., Buckley-Geer, E.J., Cooper, M.C., Hansen, S.M., High, F.W., Lin, H., Lin, Y.T., Ngeow, C.C., Rest, A., Song, J., Tucker, D., Zenteno, A., 2012. The Blanco Cosmology Survey: Data Acquisition, Processing, Calibration, Quality Diagnostics, and Data Release. ApJ 757, 83. 1204.1210.

Desai, S., Kahya, E.O., 2016. Galactic one-way Shapiro delay to PSR B1937+21. Modern Physics Letters A 31, 1650083. 1510.08228.

Detweiler, S., 1979. Pulsar timing measurements and the search for gravitational waves. ApJ 234, 1100-1104.

Devine, T.R., Goseva-Popstojanova, K., McLaughlin, M., 2016. Detection of dispersed radio pulses: a machine learning approach to candidate identification and classification. MNRAS 459, 1519-1532. 1603.09461.

Eatough, R.P., Molkenthin, N., Kramer, M., Noutsos, A., Keith, M.J., Stappers, B.W., Lyne, A.G., 2010. Selection of radio pulsar candidates using artificial neural networks. MNRAS 407, 2443-2450. 1005.5068.

Elorrieta, F., Eyheramendy, S., Jordán, A., Dékány, I., Catelan, M., Angeloni, R., Alonso-García, J., Contreras-Ramos, R., Gran, F., Hajdu, G., Espinoza, N., Saito, R.K., Minniti, D., 2016. A machine learned classifier for RR Lyrae in the VVV survey. A\&A 595, A82. 1610.05707.

Faucher-Giguère, C.A., Kaspi, V.M., 2006. Birth and Evolution of Isolated Radio Pulsars. ApJ 643, 332-355. astro-ph/0 512585.

Fawcett, T., 2006. An introduction to roc analysis. Pattern Recognition Letters 27, 861 - 874. ROC Analysis in Pattern Recognition.

Frank, E., Witten, I., 1998. Machine learning: proceedings of the fifteenth international conference. Madison, Wisconsin , 144.

Freund, Y., Schapire, R.E., 1995. A desicion-theoretic generalization of online learning and an application to boosting, in: European conference on computational learning theory, Springer. pp. 23-37.

Friedman, J.H., 2001. Greedy function approximation: A gradient boosting machine. Ann. Statist. 29, 1189-1232.

Hanley, J.A., McNeil, B.J., 1983. A method of comparing the areas under receiver operating characteristic curves derived from the same cases. Radiology 148, 839-843. PMID: 6878708. https://doi.org/10.1148/ radiology.148.3.6878708.

Hastie, T., Tibshirani, R., Friedman, J., 2001. The Elements of Statistical Learning. Springer.

He, D., Rish, I., Haws, D., Teyssedre, S., Karaman, Z., Parida, L., 2013. MINT: Mutual Information based Transductive Feature Selection for Genetic Trait Prediction. ArXiv e-prints 1310.1659.

He, H., Garcia, E.A., 2009. Learning from imbalanced data. IEEE Transactions on Knowledge and Data Engineering 21, 1263-1284.

Hewish, A., Bell, S.J., Pilkington, J.D.H., Scott, P.F., Collins, R.A., 1968. Observation of a Rapidly Pulsating Radio Source. Nature 217, 709-713.

Hinton, G.E., Salakhutdinov, R.R., 2009. Replicated softmax: an undirected topic model, in: Advances in neural information processing systems, pp. 1607-1614.

Hogden, J., Vander Wiel, S., Bower, G.C., Michalak, S., Siemion, A., Werthimer, D., 2012. Comparison of Radio-frequency Interference Mitigation Strategies for Dispersed Pulse Detection. ApJ 747, 141. 1201.1525.

Hoyle, B., Rau, M.M., Zitlau, R., Seitz, S., Weller, J., 2015. Feature importance for machine learning redshifts applied to sdss galax- ies. Monthly Notices of the Royal Astronomical Society 449, 12751283. http://mnras.oxfordjournals.org/content/449/2/ 1275. full.pdf html.

Johnston, S., Karastergiou, A., 2017. Pulsar braking and the P-dot $\{\mathrm{P}\}$ diagram. MNRAS 467, 3493-3499. 1702.03616.

Kaspi, V.M., Kramer, M., 2016. Radio Pulsars: The Neutron Star Population and Fundamental Physics. ArXiv e-prints 1602.07738.

Keith, M.J., Eatough, R.P., Lyne, A.G., Kramer, M., Possenti, A., Camilo, F., Manchester, R.N., 2009. Discovery of 28 pulsars using new techniques for sorting pulsar candidates. MNRAS 395, 837-846. 0901.3570.

Keith, M.J., Jameson, A., van Straten, W., Bailes, M., Johnston, S., Kramer, M., Possenti, A., Bates, S.D., Bhat, N.D.R., Burgay, M., Burke-Spolaor, S., D’Amico, N., Levin, L., McMahon, P.L., Milia, S., Stappers, B.W., 2010. The High Time Resolution Universe Pulsar Survey - I. System configuration and initial discoveries. MNRAS 409, 619-627. 1006.5744

Kingma, D.P., Ba, J., 2014. Adam: A method for stochastic optimization. CoRR abs/1412.6980.

Kramer, M., Stappers, B., 2015. Pulsar Science with the SKA. Advancing Astrophysics with the Square Kilometre Array (AASKA14), 361507. 04423.

Kraskov, A., Stögbauer, H., Grassberger, P., 2004. Estimating mutual information. Phys. Rev. E 69, 066138.

Lee, K.J., Guillemot, L., Yue, Y.L., Kramer, M., Champion, D.J., 2012. Application of the Gaussian mixture model in pulsar astronomy - pulsar classification and candidates ranking for the Fermi 2FGL catalogue. MNRAS 424, 2832-2840. 1205.6221.

Lorimer, D.R., Bailes, M., McLaughlin, M.A., Narkevic, D.J., Crawford, F., 2007. A Bright Millisecond Radio Burst of Extragalactic Origin. Science 318, 777. 0709.4301

Lorimer, D.R., Kramer, M., 2004. Handbook of Pulsar Astronomy.

Lyon, R., Brooke, J., Knowles, J., Stappers, B., 2013. A study on classification in imbalanced and partially-labelled data streams, in: Systems, Man, and Cybernetics (SMC), 2013 IEEE International Conference on, IEEE. pp. 1506-1511.

Lyon, R.J., Brooke, J.M., Knowles, J.D., Stappers, B.W., 2014. Hellinger Distance Trees for Imbalanced Streams. ArXiv e-prints 1405.2278.

Lyon, R.J., Stappers, B.W., Cooper, S., Brooke, J.M., Knowles, J.D., 2016. Fifty Years of Pulsar Candidate Selection: From simple filters to a new principled real-time classification approach. MNRAS 459. 1603.05166.

Maas, A.L., Hannun, A.Y., Ng, A.Y., 2013. Rectifier nonlinearities improve neural network acoustic models, in: in ICML Workshop on Deep Learning for Audio, Speech and Language Processing.

Macedo, F., Rosário Oliveira, M., Pacheco, A., Valadas, R., 2017. A theoretical framework for evaluating forward feature selection methods based on mutual information. ArXiv e-prints 1701.07761.

Manchester, R.N., Hobbs, G.B., Teoh, A., Hobbs, M., 2005. The Australia Telescope National Facility Pulsar Catalogue. AJ 129, 1993-2006. astro-ph/0412641.

Mantas, C., Puche, J., Mantas, J., 2006. Extraction of similarity based fuzzy rules from artificial neural networks. International Journal of Approximate Reasoning 43, $202-221$.

Mayr, A., Binder, H., Gefeller, O., Schmid, M., 2014. The Evolution of Boosting Algorithms - From Machine Learning to Statistical Modelling. ArXiv e-prints 1403.1452.

McLaughlin, M.A., Lyne, A.G., Lorimer, D.R., Kramer, M., Faulkner, A.J., Manchester, R.N., Cordes, J.M., Camilo, F., Possenti, A., Stairs, I.H., Hobbs, G., D’Amico, N., Burgay, M., O’Brien, J.T., 2006. Transient radio bursts from rotating neutron stars. Nature 439, 817-820. astro-ph/ 0511587.

Michalski, R.S., Carbonell, J.G., Mitchell, T.M., 2013. Machine learning: An artificial intelligence approach. Springer Science \& Business Media.

Mirabal, N., Charles, E., Ferrara, E.C., Gonthier, P.L., Harding, A.K., SánchezConde, M.A., Thompson, D.J., 2016. 3FGL Demographics Outside the Galactic Plane using Supervised Machine Learning: Pulsar and Dark Matter Subhalo Interpretations. ApJ 825, 69. 1605.00711.

Mitchell, R., Frank, E., 2017. Accelerating the xgboost algorithm using gpu computing. PeerJ Computer Science 3, e127.

Möller, A., Ruhlmann-Kleider, V., Leloup, C., Neveu, J., PalanqueDelabrouille, N., Rich, J., Carlberg, R., Lidman, C., Pritchet, C., 2016. Photometric classification of type Ia supernovae in the SuperNova Legacy Survey with supervised learning. J. Cosmology Astropart. Phys. 12, 008. 
1608.05423

Morello, V., Barr, E.D., Bailes, M., Flynn, C.M., Keane, E.F., van Straten, W., 2014. SPINN: a straightforward machine learning solution to the pulsar candidate selection problem. MNRAS 443, 1651-1662. 1406.3627.

Ng, C., 2013. Conducting The Deepest All-Sky Pulsar Survey Ever: The All-Sky High Time Resolution Universe Survey. IAU Symp. 291, 53-56. 1401.2826 .

Pedregosa, F., Varoquaux, G., Gramfort, A., Michel, V., Thirion, B., Grisel, O. Blondel, M., Prettenhofer, P., Weiss, R., Dubourg, V., et al., 2011. Scikitlearn: Machine learning in python. Journal of Machine Learning Research 12, 2825-2830.

Peng, H., Long, F., Ding, C., 2005. Feature selection based on mutual information: Criteria of max-dependency, max-relevance, and min-redundancy. IEEE Trans. Pattern Anal. Mach. Intell. 27, 1226-1238.

Ransom, S.M., 2013. Pulsars are cool. Seriously., in: van Leeuwen, J. (Ed.), Neutron Stars and Pulsars: Challenges and Opportunities after 80 years, pp. 3-10. 1211.3138.

Sadeh, I., Abdalla, F.B., Lahav, O., 2016. ANNz2: Photometric Redshift and Probability Distribution Function Estimation using Machine Learning. PASP 128, 104502. 1507.00490.

Salzberg, S.L., 1994. C4. 5: Programs for machine learning by j. ross quinlan. morgan kaufmann publishers, inc., 1993. Machine Learning 16, 235-240.

Sammut, C., Webb, G.I., 2011. Encyclopedia of Machine Learning. Springer Publishing Company, Incorporated. 1st edition.

Sevilla-Noarbe, I., Etayo-Sotos, P., 2015. Effect of training characteristics on object classification: An application using Boosted Decision Trees. Astronomy and Computing 11, 64-72. 1504.06776.

Smith, K.M., 2016. New algorithms for radio pulsar search. ArXiv e-prints 1610.06831.

Smithbauer, D.P., 2013. Analysis and Application of Automated Methods for Detecting Pulsars in the Green Bank Telescope 350MHz Drift-Scan Survey. West Virginia University.

Tamayo, D., Silburt, A., Valencia, D., Menou, K., Ali-Dib, M., Petrovich, C., Huang, C.X., Rein, H., van Laerhoven, C., Paradise, A., Obertas, A., Murray, N., 2016. A Machine Learns to Predict the Stability of Tightly Packed Planetary Systems. ApJ 832, L22. 1610.05359.

Taylor, Jr., J.H., 1994. Binary pulsars and relativistic gravity. Reviews of Modern Physics 66, 711-719.

The IceCube Collaboration, Aartsen, M.G., Abraham, K., Ackermann, M., Adams, J., Aguilar, J.A., Ahlers, M., Ahrens, M., Altmann, D., Anderson, T., et al., 2015. The IceCube Neutrino Observatory - Contributions to ICRC 2015 Part II: Atmospheric and Astrophysical Diffuse Neutrino Searches of All Flavors. ArXiv e-prints 1510.05223.

Tramacere, A., Paraficz, D., Dubath, P., Kneib, J.P., Courbin, F., 2016. ASTErIsM: application of topometric clustering algorithms in automatic galaxy detection and classification. MNRAS 463, 2939-2957. 1609.06728.

Wagstaff, K.L., Tang, B., Thompson, D.R., Khudikyan, S., Wyngaard, J., Deller, A.T., Palaniswamy, D., Tingay, S.J., Wayth, R.B., 2016. A Machine Learning Classifier for Fast Radio Burst Detection at the VLBA. PASP 128, 084503. 1606.08605 .

Wolszczan, A., Frail, D.A., 1992. A planetary system around the millisecond pulsar PSR1257 + 12. Nature 355, 145-147.

Yusifov, I., Küçük, I., 2004. Revisiting the radial distribution of pulsars in the Galaxy. A\&A 422, 545-553. astro-ph/ 0405559.

Zhang, H., 2004. The optimality of naive bayes, in: Barr, V., Markov, Z. (Eds.), Proceedings of the Seventeenth International Florida Artificial Intelligence Research Society Conference (FLAIRS 2004), AAAI Press.

Zhu, W.W., Berndsen, A., Madsen, E.C., Tan, M., Stairs, I.H., Brazier, A. Lazarus, P., Lynch, R., Scholz, P., Stovall, K., Ransom, S.M., Banaszak, S., Biwer, C.M., Cohen, S., Dartez, L.P., Flanigan, J., Lunsford, G., Martinez, J.G., Mata, A., Rohr, M., Walker, A., Allen, B., Bhat, N.D.R., Bogdanov, S., Camilo, F., Chatterjee, S., Cordes, J.M., Crawford, F., Deneva, J.S., Desvignes, G., Ferdman, R.D., Freire, P.C.C., Hessels, J.W.T., Jenet, F.A., Kaplan, D.L., Kaspi, V.M., Knispel, B., Lee, K.J., van Leeuwen, J., Lyne, A.G., McLaughlin, M.A., Siemens, X., Spitler, L.G., Venkataraman, A., 2014. Searching for Pulsars Using Image Pattern Recognition. ApJ 781, 117. 1309.0776.

Zitlau, R., Hoyle, B., Paech, K., Weller, J., Rau, M.M., Seitz, S., 2016. Stacking for machine learning redshifts applied to SDSS galaxies. MNRAS 460, 3152-3162. 1602.06294. 